\title{
The Numerical Method for Solving Differential Equations of Lane-Emden Type by Padé Approximation
}

\author{
Muhammed Yiğider, ${ }^{1}$ Khatereh Tabatabaei, $^{2}$ and Ercan Çelik ${ }^{3}$ \\ ${ }^{1}$ Department of Mathematics, Faculty of Art and Science, Erzincan University, 24100 Erzincan, Turkey \\ ${ }^{2}$ Department of Mathematics, Young Researchers Club, Azad University, 55518/134 Bonab, Iran \\ ${ }^{3}$ Department of Mathematics, Faculty of Science, Atatürk University, 25240 Erzurum, Turkey
}

Correspondence should be addressed to Ercan Çelik, ercelik@atauni.edu.tr

Received 13 January 2011; Accepted 22 February 2011

Academic Editor: Ibrahim Yalcinkaya

Copyright (C) 2011 Muhammed Yiğider et al. This is an open access article distributed under the Creative Commons Attribution License, which permits unrestricted use, distribution, and reproduction in any medium, provided the original work is properly cited.

Numerical solution differential equation of Lane-Emden type is considered by Padé approximation. We apply these method to two examples. First differential equation of Lane-Emden type has been converted to power series by one-dimensional differential transformation, then the numerical solution of equation was put into Padé series form. Thus, we have obtained numerical solution differential equation of Lane-Emden type.

\section{Introduction}

Lane-Emden equations have the following form [1-4]:

$$
\begin{gathered}
y^{\prime \prime}+\frac{k}{x} y^{\prime}+f(x, y)=g(x), \quad 0 \leq x \leq 1, k \geq 0, \\
y(0)=A, \quad y^{\prime}(0)=B
\end{gathered}
$$

where $A$ and $B$ are constants, $f(x, y)$ is a continuous real-valued function, and $g(x) \in$ $C[0,1]$. Lane-Emden equations are singular initial value problems relating to second-order ordinary differential equations (ODEs) which have been used to model several phenomena in mathematical physics and astrophysics such as thermal explosions [5], the thermal behavior of a spherical cloud of gas, isothermal gas spheres, and thermionic currents [5]. Several 
methods for the solutions of Lane-Emden equations have been presented. Wazwaz [6] has given a general way to construct exact and series solutions to Lane-Emden equations by employing the Adomian decomposition method. A numerical solution of Lane-Emden equations is given based on the Legendre wavelets methods [4]. The variational iteration method is used to solve differential equations arising in astrophysics including the LaneEmden equation $[7,8]$. The homotopy perturbation method and the integral operator method were suggested in [9-11].

In this paper, we apply these method to two examples. First, differential equation of Lane-Emden type has been converted to power series by one-dimensional differential transformation; differential transformation was introduced first by Zhou [12]. Then the numerical solution of equation was put into Padé series form [13]. The Padé approximation method was used to accelerate the convergence of the power series solution. Thus, we obtain numerical solution differential equation of Lane-Emden type.

\section{One-Dimensional Differential Transform}

Differential transform of function $y(x)$ is defined as follows:

$$
Y(k)=\frac{1}{k !}\left[\frac{d^{k} y(x)}{d x^{k}}\right]_{x=0} .
$$

In (2.1), $y(x)$ is the original function and $Y(k)$ is the transformed function, which is called the T-function. Differential inverse transform of $Y(k)$ is defined as

$$
y(x)=\sum_{k=0}^{\infty} x^{k} Y(k)
$$

From (2.1) and (2.2), we obtain

$$
y(x)=\sum_{k=0}^{\infty} \frac{x^{k}}{k !}\left[\frac{d^{k} y(x)}{d x^{k}}\right]_{x=0} .
$$

Equation (2.3) implies that the concept of differential transform is derived from Taylor series expansion, but the method does not evaluate the derivatives symbolically.

However, relative derivatives are calculated by an iterative way which is described by the transformed equations of the original functions. In this study, we use the lower case letter to represent the original function, and upper case letter represent the transformed function.

From the definitions of (2.1) and (2.2), it is easily proven that the transformed functions comply with the basic mathematics operations shown in Table 1. 
Table 1: The fundamental operations of one-dimensional differential transform method.

\begin{tabular}{lc}
\hline Original function & Transformed function \\
\hline$y(x)=u(x) \pm v(x)$ & $\Upsilon(k)=U(k) \pm V(k)$ \\
$y(x)=c w(x)$ & $\Upsilon(k)=c W(k)$ \\
$y(x)=d w / d x$ & $Y(k)=(k+1) W(k+1)$ \\
$y(x)=d^{j} w / d x^{j}$ & $Y(k)=(k+1)(k+2) \cdots(k+j) W(k+j)$ \\
$y(x)=u(x) v(x)$ & $\Upsilon(k)=\sum_{r=0}^{k} U(r) V(k-r)$ \\
$y(x)=x^{j}$ & $\Upsilon(k)=\delta(k-j)=\left\{\begin{array}{l}1, k=j \\
0, k \neq j\end{array}\right.$ \\
\hline
\end{tabular}

In actual applications, the function $y(x)$ is expressed by a finite series and (2.2) can be written as

$$
y(x)=\sum_{k=0}^{m} x^{k} Y(k)
$$

Equation (2.3) implies that $\sum_{k=m+1}^{\infty} x^{k} Y(k)$ is negligibly small. In fact, $m$ is decided by the convergence of natural frequency in this study.

Definition 2.1. If $y(x)=u(x) v(x), u(x)=D^{-1}[U(k)], v(x)=D^{-1}[V(k)]$, and $\otimes$ denote convolution. Then $[y(x)]=D[u(x) v(x)]=U(k) \otimes V(k)=\sum_{r=0}^{k} U(r) V(k-r)$.

\section{Padé Approximation}

Suppose that we are given a power series $\sum_{i=0}^{\infty} a_{i} x^{i}$, representing a function $f(x)$, so that

$$
f(x)=\sum_{i=0}^{\infty} a_{i} x^{i}
$$

A Padé approximation is a rational fraction

$$
\left[\frac{L}{M}\right]=\frac{p_{0}+p_{1} x+\cdots+p_{L} x^{L}}{q_{0}+q_{1} x+\cdots+q_{M} x^{M}}
$$

which has a Maclaurin expansion which agrees with (3.1) as much as possible. Notice that in (3.2), there are $L+1$ numerator coefficients and $M+1$ denominator coefficients. There is a more or less irrelevant common factor between them, and for definiteness, we take $q_{0}=1$. This choice turns out to be an essential part of the precise definition, and (3.2) is our conventional notation with this choice for $q_{0}$. So, there are $L+1$ independent numerator coefficients and $M$ independent numerator coefficients, making $L+M+1$ unknown coefficients in all. This number suggests that normally the $[L / M]$ ought to fit the power series (3.1) through the 
orders $1, x, x^{2}, \ldots, x^{L+M}$ in the notation of formal power series

$$
\sum_{i=0}^{\infty} a_{i} x^{i}=\frac{p_{0}+p_{1} x+\cdots+p_{L} x^{L}}{q_{0}+q_{1} x+\cdots+q_{M} x^{M}}+O\left(x^{L+M+1}\right) .
$$

Multiply both sides of (3.3) by the denominator of right side in (3.3), and compare the coefficients of both sides of (3.3), then we have

$$
\begin{gathered}
a_{l}+\sum_{k=1}^{m} a_{l-k} q_{k}=p_{l}, \quad(l=0, \ldots, M), \\
a_{l}+\sum_{k=1}^{L} a_{l-k} q_{k}=0, \quad(l=M+1, \ldots, M+L) .
\end{gathered}
$$

Solve the linear equation in (3.5), then we have $q_{k}(k=1, \ldots, L)$. And substitute $q_{k}$ into (3.4), then we have $p_{1}(l=0, \ldots, M)$. Therefore, we have constructed a $[L / M]$ Padé approximation, which agrees with $\sum_{i=0}^{\infty} a_{i} x^{i}$ through order $x^{L+M}$. If $M \leq L \leq M+2$, where $M$ and $L$ are the degree of numerator and denominator in Padé series, respectively, then Padé series gives an A-stable formula for an ordinary differential equation.

\section{Numerical Examples}

Example 4.1 (see Table 2 and Figure 1).

$$
\begin{aligned}
u^{\prime \prime}(x)+\frac{2}{x} u^{\prime}(x) & =2\left(2 x^{2}+3\right) u(x), \quad 0 \leq x \leq 1, \\
u(0) & =1, \quad u^{\prime}(0)=0
\end{aligned}
$$

where the exact solution is $u(x)=e^{x^{2}}$. Multiplying both sides of (4.1) by $x$,

$$
x u^{\prime \prime}(x)+2 u^{\prime}(x)=4 x^{3} u(x)+6 x u(x), \quad 0 \leq x \leq 1 .
$$

By using the fundamental operations of one-dimensional differential transform method in Table 1, we obtained following recurrence relation:

$$
\delta(k-1) \otimes(k+1)(k+2) U(k+2)+2(k+1) U(k+1)=4 \delta(k-3) \otimes U(k)+6 \delta(k-1) \otimes U(k) .
$$


Table 2: Numerical solution of $u(x)$.

\begin{tabular}{lccc}
\hline$x$ & $u(x)$ & $P[5 / 4]$ & $|u(x)-P[5 / 4]|$ \\
\hline 0.1 & 1.010050167 & 1.010050167 & 0 \\
0.2 & 1.040810774 & 1.040810774 & 0 \\
0.3 & 1.094174234 & 1.094174275 & $4.1 \times 10^{-8}$ \\
0.4 & 1.173509974 & 1.173510700 & $7.26 \times 10^{-7}$ \\
0.5 & 1.284023668 & 0.000006741 \\
0.6 & 1.284016927 & 1.43317285 & 0.000041445 \\
0.7 & 1.433275840 & 1.632251266 & 0.000191099 \\
0.8 & 1.632060167 & 1.896191187 & 0.000710013 \\
0.9 & 1.895481174 & 2.246777235 & 0.002217601 \\
1.0 & 2.244559634 & 2.714285714 & 0.005952380 \\
\hline
\end{tabular}

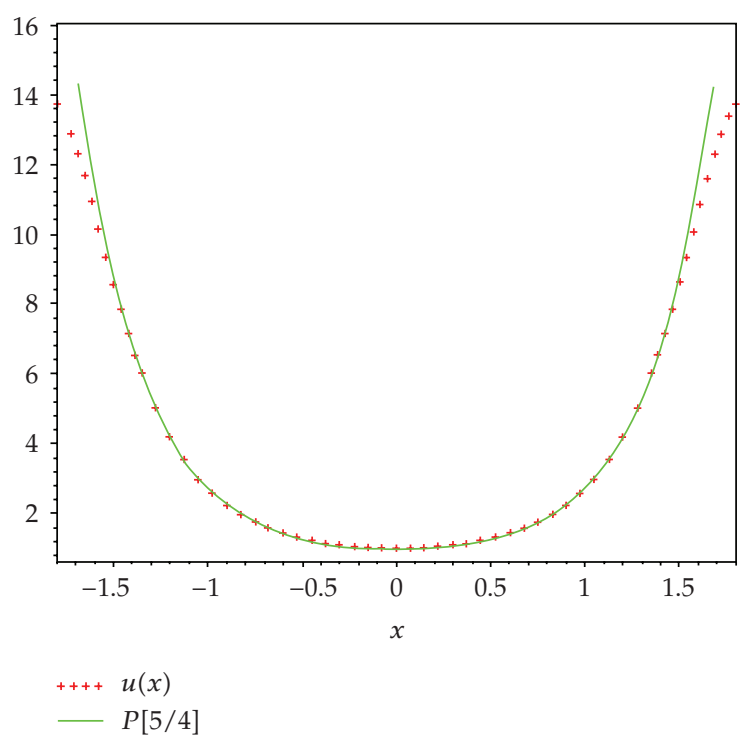

Figure 1: Values of $u(x)$ and its $P[5 / 4]$ Padé approximant.

Then,

$$
\begin{gathered}
\sum_{l=0}^{k} \delta(l-1)(k-l+1)(k-l+2) U(k-l+2)+2(k+1) U(k+1) \\
=4 \sum_{l=0}^{k} \delta(l-3) U(k-l)+\sum_{l=0}^{k} 6 \delta(l-1) U(k-l), \\
U(0)=1, \quad U(1)=0, \quad U(2)=1, \quad U(3)=0, \quad U(4)=\frac{1}{2}, \quad U(5)=0,
\end{gathered}
$$




$$
\begin{gathered}
U(6)=\frac{1}{6}, \quad U(7)=0, \quad U(8)=\frac{1}{24}, \quad U(9)=0, \ldots, \\
u^{*}(x)=U(0)+x U(1)+x^{2} U(2)+x^{3} U(3)+\ldots, \\
u^{*}(x)=1+x^{2}+\frac{1}{2} x^{4}+\frac{1}{6} x^{6}+\frac{1}{24} x^{8}+\cdots=1+x^{2}+\frac{1}{2} x^{4}+\frac{1}{3 !} x^{6}+\frac{1}{4 !} x^{8}+\cdots+\frac{1}{n !} x^{2 n} .
\end{gathered}
$$

Power series $u^{*}(x)$ can be transformed into Padé series

$$
P\left[\frac{5}{4}\right]=\frac{\left(1+(1 / 2) x^{2}+(1 / 12) x^{4}\right)}{\left(1-(1 / 2) x^{2}+(1 / 12) x^{4}\right)}=\frac{\left(1+0.5 x^{2}+0.83333333333 x^{4}\right)}{\left(1-0.5 x^{2}+0.83333333333 x^{4}\right)} .
$$

Example 4.2 (see Table 3 and Figure 2). Consider the Lane-Emden-type equation

$$
y^{\prime \prime}+\frac{2}{x} y^{\prime}+\left(8 e^{y}+4 e^{y / 2}\right)=0,
$$

with initial values

$$
y(0)=0, \quad y^{\prime}(0)=0,
$$

where the exact solution is $y(x)=-2 \ln \left(1+x^{2}\right)$.

Multiplying both sides of equation by $x$,

$$
x y^{\prime \prime}+2 y^{\prime}+8 x e^{y}+4 x e^{y / 2}=0 .
$$

Also, $e^{y}$ and $e^{y / 2}$ are defined as follows:

$$
e^{y}=1+y, \quad e^{y / 2}=1+\frac{y}{2}
$$

Then,

$$
x y^{\prime \prime}+2 y^{\prime}+12 x+10 x y=0 .
$$

By using the fundamental operations of one-dimensional differential transform method in Table 1, we obtain the following recurrence relation:

$$
\delta(k-1) \otimes(k+1)(k+2) Y(k+2)+2(k+1) Y(k+1)+12 \delta(k-1)+10 \delta(k-1) \otimes Y(k)=0
$$


Table 3: Numerical solution of $y(x)$.

\begin{tabular}{lccc}
\hline$x$ & $y(x)$ & $P[5 / 4]$ & $|y(x)-P[5 / 4]|$ \\
\hline 0.1 & -0.01990023712 & -0.01990023713 & $1 \times 10^{-11}$ \\
0.2 & -0.07841498810 & -0.07841499080 & $2.70 \times 10^{-9}$ \\
0.3 & -0.1720671642 & -0.1720673162 & $1.520 \times 10^{-7}$ \\
0.4 & -0.2953112381 & -0.2953138699 & 0.0000026318 \\
0.5 & -0.4408387684 & -0.4408625091 & 0.0000237407 \\
0.6 & -0.5998683214 & -0.6000097952 & 0.0001414738 \\
0.7 & -0.7622819782 & -0.7629141475 & 0.0006321693 \\
0.8 & -0.9164312381 & -0.9187160286 & 0.0022847905 \\
0.9 & -1.048395758 & -1.055408536 & 0.007012778 \\
1.0 & -1.140438988 & -1.159333345 & 0.018894357 \\
\hline
\end{tabular}

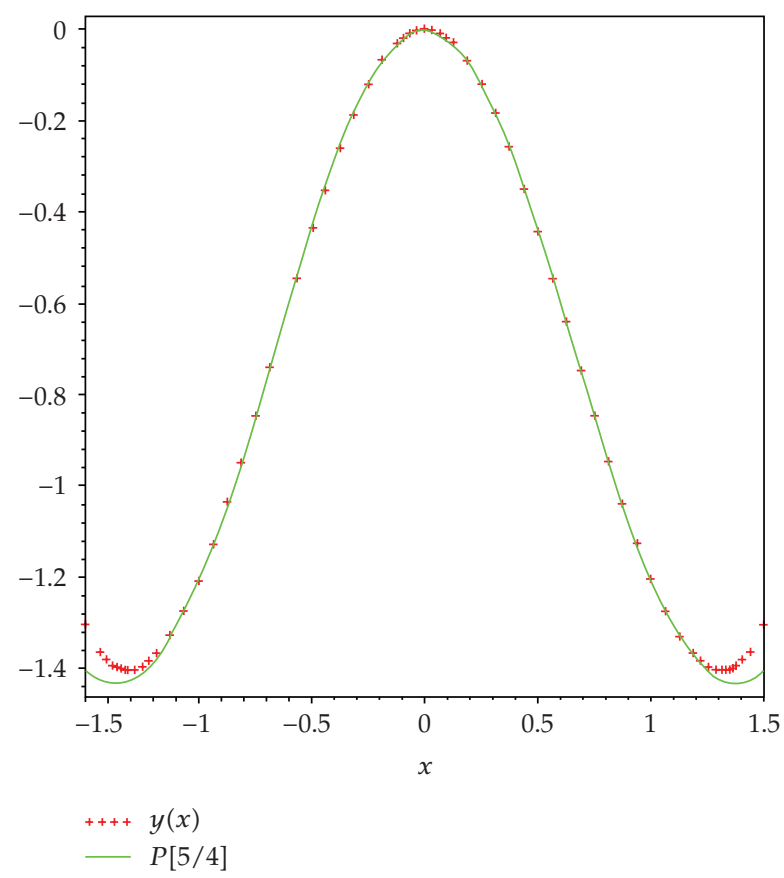

Figure 2: Values of $y(x)$ and its $P[5 / 4]$ Padé approximant.

Then,

$$
\begin{aligned}
& \sum_{l=0}^{k} \delta(l-1)(k-l+1)(k-l+2) Y(k-l+2)+2(k+1) Y(k+1)+12 \delta(k-1) \\
& \quad+10 \sum_{l=0}^{k} \delta(l-1) Y(k-l)=0, \\
& y(0)=0, \quad y(1)=0, \quad y(2)=-2, \quad y(3)=0, \quad y(4)=1, \quad y(5)=0,
\end{aligned}
$$




$$
\begin{gathered}
y(6)=-\frac{5}{21}, \quad y(7)=0, \quad y(8)=\frac{25}{756}, \ldots, \\
y^{*}(x)=y(0)+x y(1)+x^{2} y(2)+x^{3} y(3)+\ldots, \\
y^{*}(x)=-2 x^{2}+x^{4}-\frac{5}{21} x^{6}+\frac{25}{756} x^{8}+\ldots
\end{gathered}
$$

Power series $y^{*}(x)$ can be transformed into Padé series

$$
\begin{aligned}
P\left[\frac{5}{4}\right] & =\frac{\left((589 / 704) x^{4}-2 x^{2}\right)}{\left(1+(115 / 1408) x^{2}-(4625 / 59136) x^{4}\right)} \\
& =\frac{\left(0.8366477273 x^{4}-2 x^{2}\right)}{\left(1+0.08167613636 x^{2}-0.07820955087 x^{4}\right)} .
\end{aligned}
$$

\section{Conclusion}

A Padé approximation method has proposed for solving differential equation of LaneEmden type in this study. This method is very simple and effective for most of Lane-Emden equations. Numerical results explicitly reveal the complete reliability of the proposed Padé approximation.

\section{References}

[1] A. Aslanov, "Determination of convergence intervals of the series solutions of Emden-Fowler equations using polytropes and isothermal spheres," Physics Letters A, vol. 372, no. 20, pp. 3555-3561, 2008.

[2] A. Aslanov, "Approximate solutions of Emden-Fowler type equations," International Journal of Computer Mathematics, vol. 86, no. 5, pp. 807-826, 2009.

[3] E. Momoniat and C. Harley, "Approximate implicit solution of Lane-Emden equation," New Astronomy, vol. 11, pp. 520-526, 2006.

[4] S. A. Yousefi, "Legendre wavelets method for solving differential equations of Lane-Emden type," Applied Mathematics and Computation, vol. 181, no. 2, pp. 1417-1422, 2006.

[5] O. U. Richardson, The Emission of Electricity from Hot Bodies, Longmans Green and Company, London, UK, 1921.

[6] A.-M. Wazwaz, "A new algorithm for solving differential equations of Lane-Emden type," Applied Mathematics and Computation, vol. 118, no. 2-3, pp. 287-310, 2001.

[7] M. Dehghan and F. Shakeri, "Approximate solution of a differential equation arising in astrophysics using the variational iteration method," New Astronomy, vol. 13, no. 1, pp. 53-59, 2008.

[8] A. Yıldırım and T. Öziş, "Solutions of singular IVPs of Lane-Emden type by the variational iteration method," Nonlinear Analysis. Theory, Methods \& Applications, vol. 70, no. 6, pp. 2480-2484, 2009.

[9] J. I. Ramos, "Series approach to the Lane-Emden equation and comparison with the homotopy perturbation method," Chaos, Solitons and Fractals, vol. 38, no. 2, pp. 400-408, 2008.

[10] S. K. Vanani and A. Aminataei, "On the numerical solution of differential equations of Lane-Emden type," Computers \& Mathematics with Applications, vol. 59, no. 8, pp. 2815-2820, 2010.

[11] A. Yıldırım and T. Öziş, "Solutions of singular IVPs of Lane-Emden type by homotopy perturbation method," Physics Letters. Section A, vol. 369, no. 1-2, pp. 70-76, 2007. 
[12] J. K. Zhou, Differential Transformation and Its Application for Electrical Circuits, Huazhong University Press, Wuhan, China, 1986.

[13] E. Çelik and M. Bayram, "Arbitrary order numerical method for solving differential-algebraic equation by Padé series," Applied Mathematics and Computation, vol. 137, no. 1, pp. 57-65, 2003. 


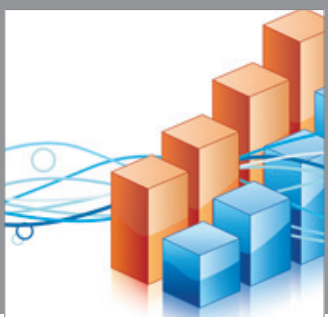

Advances in

Operations Research

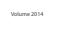

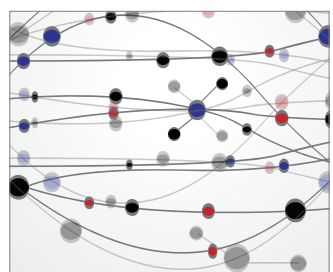

\section{The Scientific} World Journal
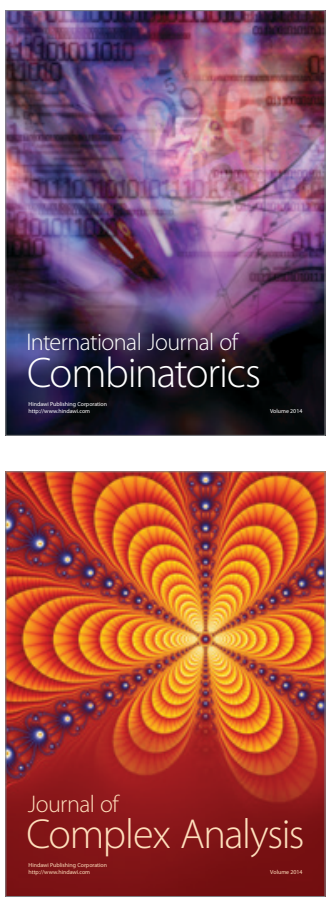

International Journal of

Mathematics and

Mathematical

Sciences
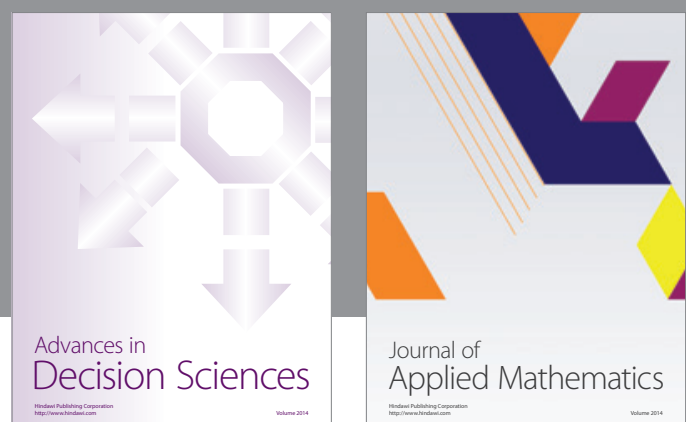

Journal of

Applied Mathematics
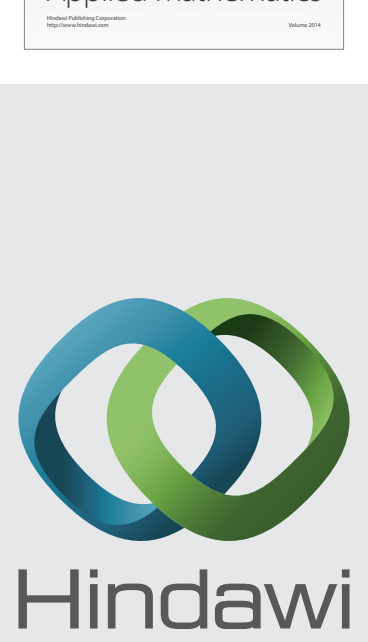

Submit your manuscripts at http://www.hindawi.com
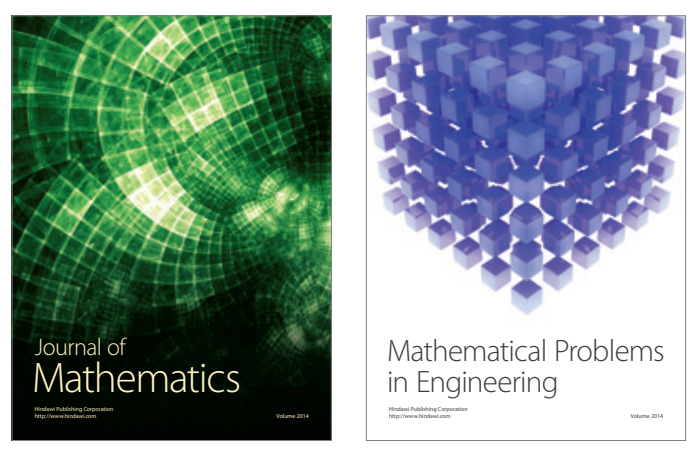

Mathematical Problems in Engineering
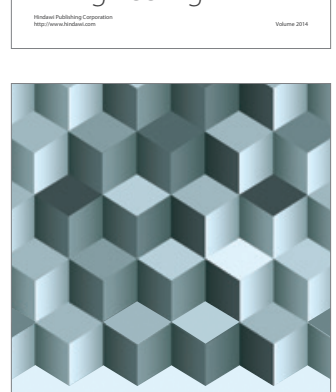

Journal of

Function Spaces
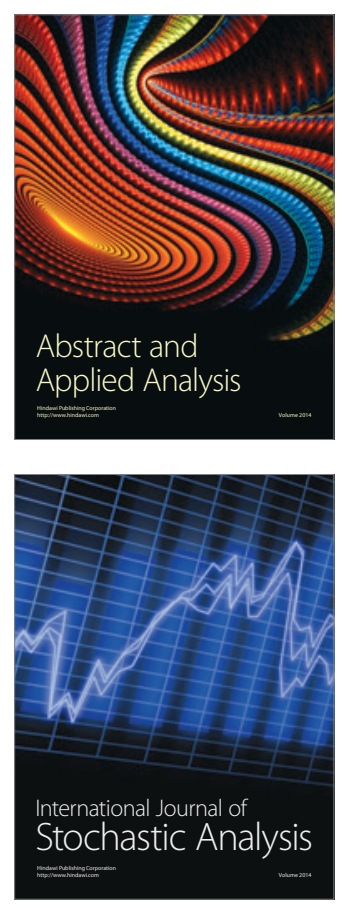

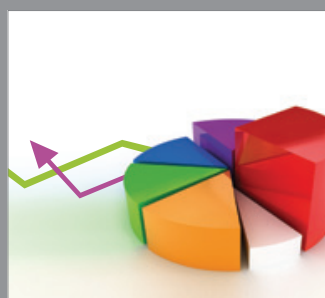

ournal of

Probability and Statistics

Promensencen
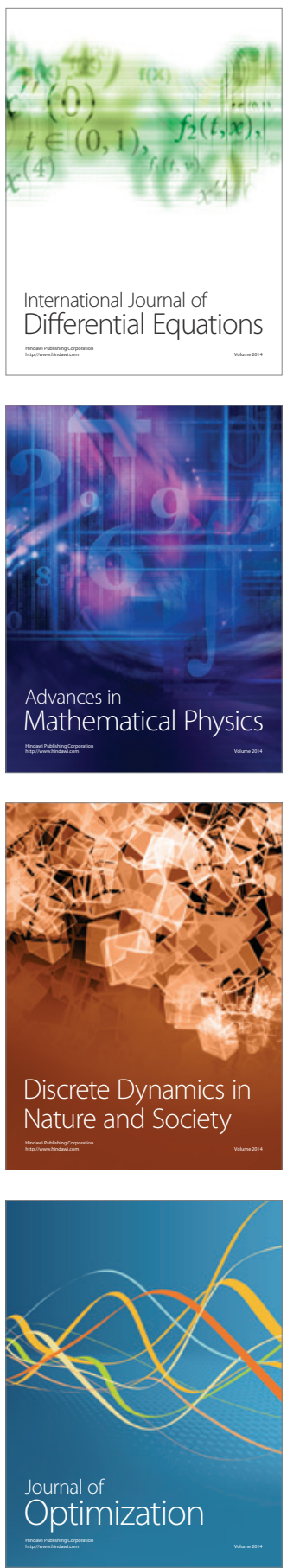